\title{
Limits on ultra-high energy photons with the Pierre Auger Observatory
}

\author{
Julian Rautenberg ${ }^{* a}$ for the Pierre Auger Collaboration ${ }^{\dagger b}$ \\ ${ }^{a}$ Bergische Universität Wuppertal, Wuppertal, Germany \\ ${ }^{b}$ Observatorio Pierre Auger, Av. San Martín Norte 304, 5613 Malargüe, Argentina \\ E-mail: auger_spokespersons@ @ fnal.gov \\ Full author list: http://www.auger.org/archive/authors_icrc_2019_.html
}

\begin{abstract}
The Pierre Auger Observatory is the most sensitive air-shower detector for primary photons with energies above $\sim 0.2 \mathrm{EeV}$. Using the Surface Detector array (SD), photons in the zenith angle range from $30^{\circ}$ to $60^{\circ}$ can be identified through the broad time-structure of the signals expected to be induced in the SD stations. An additional signature for photon-induced air showers is the steeper lateral distribution of secondary particles at the ground with respect to nucleonic showers. Stringent limits are set to the diffuse flux of ultra-high energy (UHE) photons above $10 \mathrm{EeV}$, using SD data collected between 2004 and mid-2018 with an exposure of $40000 \mathrm{~km}^{2} \mathrm{sr}$ yr. Below $1 \mathrm{EeV}$, unprecedented separation power between primary photons and hadrons can be achieved by combining observables from the low-energy enhancements of the Pierre Auger Observatory, namely three upwards-pointing fluorescence telescopes, which can directly measure the atmospheric depth of the shower maximum, and the $750 \mathrm{~m} \mathrm{SD}$ array, which again accesses the steeper lateral distribution of photon-induced air showers. For the first time, limits on the integral photon flux below $1 \mathrm{EeV}$ are presented using data collected by the Pierre Auger Observatory between mid-2010 and 2015. This extends the range of photon searches at the Pierre Auger Observatory to about three decades in energy.
\end{abstract}

36th International Cosmic Ray Conference -ICRC2019-

July 24th - August 1st, 2019

Madison, WI, U.S.A.

\footnotetext{
${ }^{*}$ Speaker.

${ }^{\dagger}$ for collaboration list see PoS(ICRC2019)1177
} 


\section{Introduction}

The search for ultra-high energy (UHE) photons is a key to reveal the origin of the highest energetic cosmic rays. Nuclei interacting with the extra-galactic background light, dominantly the cosmic microwave background (CMB), are expected to produce a flux of UHE photons that can propagate over several Mpc. The expected cosmogenic flux depends on the composition and energy distribution of the source emission, as well as the distribution and cosmological evolution of the sources themselves.

The Pierre Auger Observatory [1] is a hybrid detector, consisting of a Surface Detector (SD) with 1600 stations distributed over $3000 \mathrm{~km}^{2}$ in a triangular grid with a spacing of $1.5 \mathrm{~km}$, as well as a Fluorescence Detector (FD) of 27 telescopes overlooking the area from four sites from the boarder of the array. To extend the exposure to smaller energies below $10^{18} \mathrm{eV}$, an area of $23.5 \mathrm{~km}^{2}$ is equipped with 60 additional SD stations to achieve half spacing of $750 \mathrm{~m}$ and three of the FD telescopes (HEAT) have a field of view inclined by $30^{\circ}$ with respect to the standard FD telescopes above the $750 \mathrm{~m}$ array.

\section{Photon searches at the Pierre Auger Observatory}

SD observables that have the potential to separate the photon showers from the hadronic ones are based on the difference in the signals in the particle detectors due to the later shower development, measured in the depth of the shower-maximum, $X_{\max }$. For a photon, the electromagnetic shower on average has a steeper lateral fall-off and a slower rise of the signal in the SD stations on ground than hadronic showers.

Besides the use of the SD for the shower measurements on the ground, the Pierre Auger Observatory can exploit the hybrid setup and directly measure the longitudinal profile of the showers with the FD. $X_{\max }$ gives a direct and powerful estimator for the composition, thus discriminating photons from hadrons as primary cosmic ray particles. The hybrid measurements are limited by the about $15 \%$ uptime of the FD, though. Searches for photons and the derived upper limits on the flux of photons as well as on the photon fractions of the measured cosmic ray flux have been already published, using the SD data $[2,3]$ as well as hybrid ones $[4,5,6]$. In addition, the hybrid search has been extended to search for point-like sources [7] and stacking of a source catalogue [8].

This contribution presents in the next section an extension of the previous analysis based on the SD observables to the current data-set. Then the extension of the hybrid photon search towards lower energies is described.

\section{Diffuse photon flux at the highest energies}

The measurement for energies above $10 \mathrm{EeV}$ follows the one presented in [3]. The range in zenith angle used is $30^{\circ}<\theta<60^{\circ}$. The data-period has been extended, and covers the period from 1 Jan 2004 to 30 Jun 2018. The corresponding exposure for that zenith angle range has been determined to $40000 \mathrm{~km}^{2}$ sryr. To estimate the efficiency and the optimal separation, a photon signal Monte Carlo (MC) sample has been simulated using CORSIKA [9] with EPOS-LHC [10] as hadronic interaction model. The conversion of UHE phtons in the geomagnetic field is simulated 

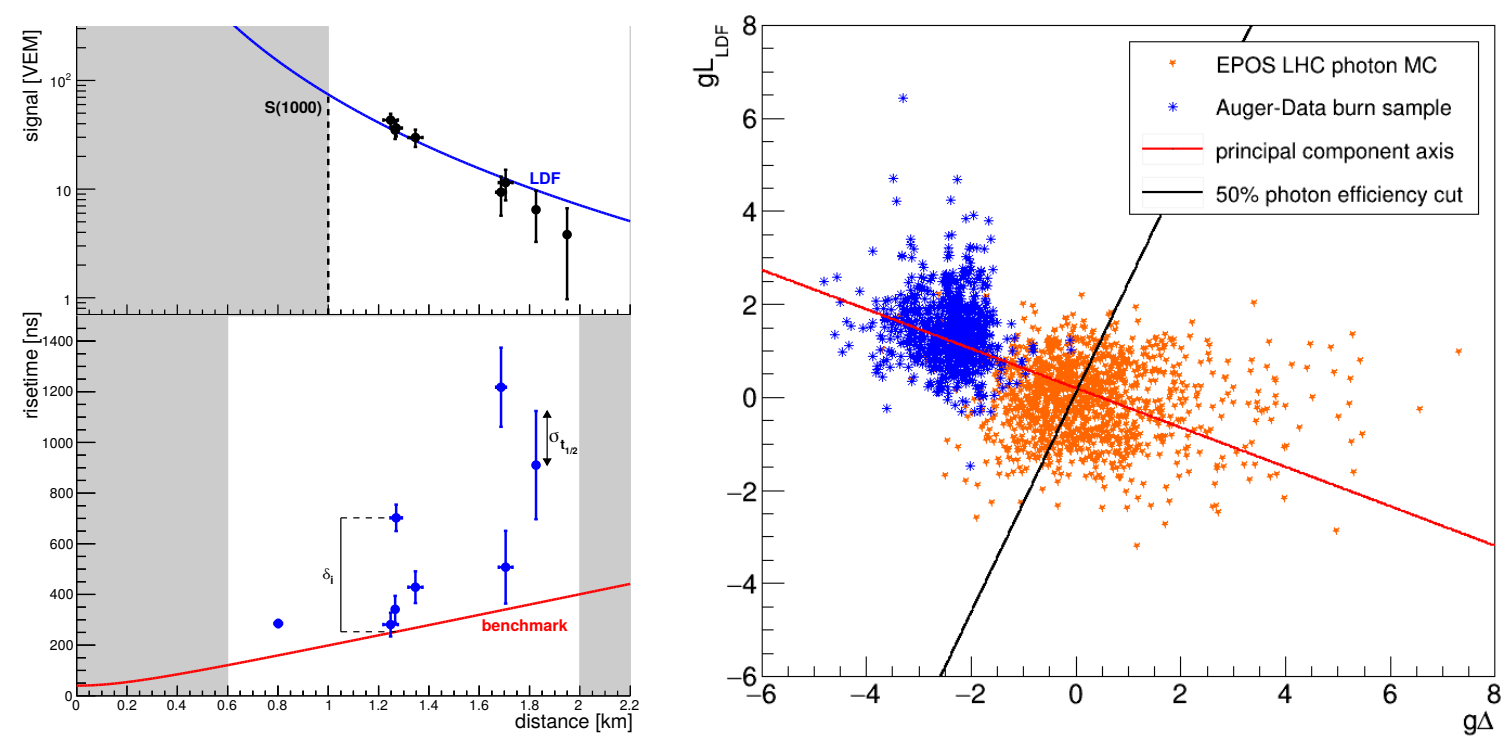

Figure 1: Left: Signal (top) and risetime of the SD stations (bottom) vs. distance to the shower axis for a simulated photon event, together with the LDF (top) and risetime-benchmark (bottom) parametrization. Right: Principal Component Analysis of the data (blue) and the MC prediction for photons (orange) for the normalized observables $g \Delta$ and $g L_{\mathrm{LDF}}$ described in the text.

using PRESHOWER [11]. The Landau-Pomeranchuk-Migdal (LPM) effect is also implemented in the simulation. The photon $\mathrm{MC}$ has been reweighted to an energy distribution according to a $E^{-2}$ spectrum. For this analysis aiming at identifying photons in the UHECR-flux we do not use any simulation of nucleonic primary cosmic rays (background $\mathrm{MC}$ sample) to avoid the large uncertainties in the tail of the distributions due to the use of hadronic interaction models. Where needed, $2 \%$ of the data are used as background sample, which are excluded from the measurement (burned). With respect to [3] the hadronic interaction model has changed, which has only a minor effect on the photon simulations. The simulation and reconstruction show minor improvement, i.e. for the data reconstruction in terms of the automatic and therefore less biased detection of the rare peculiarities in photomultipliers of the SD stations. These usually have no significant effect on the integral, and therefore signal estimation, but on the shape which is used for the composition estimation. The reconstruction of the energy is done using the observable $S_{1000}$, the signal at 1000 $\mathrm{m}$ from the shower axis estimated using a fit of a lateral distribution function parametrized to describe the Auger data. The conversion of $S_{1000}$ to the energy corresponding to a photon shower is done in an iterative procedure [12]. To avoid biases only showers which are almost fully developed are selected by requiring the $X_{\max }$ to be no more than $50 \mathrm{~g} / \mathrm{cm}^{2}$ below ground.

The selection of the photon candidates has been performed using two estimators. The first one uses the steeper lateral distribution function (LDF) of electromagnetic showers. For this, the ratio of the measured signal, $S_{i}$, and the expected signal according to the LDF fit at the distance $r_{i}$ of that $\mathrm{SD}$ station $i$ to the shower axis, $S_{\mathrm{LDF}}\left(r_{i}\right)$, is determined. The observable is the logarithm of the average of these ratios:

$$
L_{\mathrm{LDF}}=\log _{10}\left(\frac{1}{N} \sum_{i=1}^{N} \frac{S_{i}}{S_{\mathrm{LDF}}\left(r_{i}\right)}\right)
$$




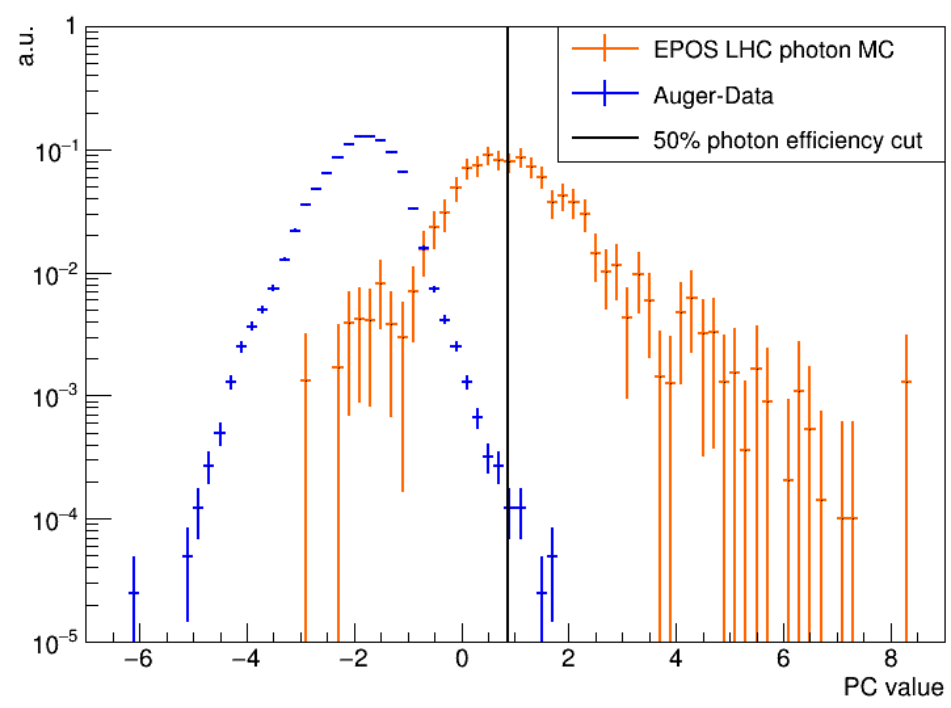

Figure 2: Distribution of the data (blue) and the MC prediction for photons (orange) along the principal component axis. For better comparison, the two distributions are normalized to an integral of one. The line indicates the signal selection at the median of the photon MC sample. 11 data events are above the threshold.

using only SD stations with $r_{i}>1000 \mathrm{~m}$. Photon candidates are expected to have smaller signals at larger distances to the shower axis, resulting in a negative $L_{\mathrm{LDF}}$. The second observable is based on the electromagnetic signal in the stations, which is on average delayed compared to the fast-rising signal of the muons. We therefore calculate the risetime, $t_{1 / 2}$, as the time between the 0.1 and the 0.5 quantile of the time trace. It should be noted, that the difference in the muonic and electromagnetic signal decreases with smaller distance of the observer to the $X_{\max }$ for pure geometrical reasons, additionally increasing the $t_{1 / 2}$ for the larger $X_{\max }$ of photon showers. The measured $t_{1 / 2}$ shows a clear asymmetry in the azimuth angle with respect to the shower direction. This asymmetry, depending also on distance to the shower axis as well as the zenith angle, can be parametrized and thus corrected for, resulting in the corrected SD station risetime, $t_{1 / 2}^{i}$. To select photon candidates, an observable is constructed from the average $t_{1 / 2}^{i}$ and its uncertainty from the data, the so called benchmark, $t_{1 / 2}^{\text {bench }}$. To obtain an event-wise observable, $\Delta$, the residuals of $t_{1 / 2}^{i}$ of the SD stations from the benchmark are averaged similarly as described in [13],

$$
\Delta=\frac{1}{N} \sum_{i=1}^{N} \frac{t_{1 / 2}^{i}-t_{1 / 2}^{\text {bench }}}{\sigma_{t_{1 / 2}}^{i}}
$$

Here, only SD stations with an observed signal of minimally $6 \mathrm{VEM}^{1}$ and a distance to the shower axis $600 \mathrm{~m}<r_{i}<2000 \mathrm{~m}$ are considered. The deviation from the average LDF leading to $L_{\mathrm{LDF}}$ and the deviation of the risetime from the benchmark leading to $\Delta$ are illustrated for a simulated photon event in Fig. 1 left.

To obtain an optimal discrimination, the two observables are transformed using a Principal Component Analysis (PCA). For this, the observables are normalized for the photon MC sample so

\footnotetext{
${ }^{1}$ the signal as calibrated to a vertical equivalent muon
} 

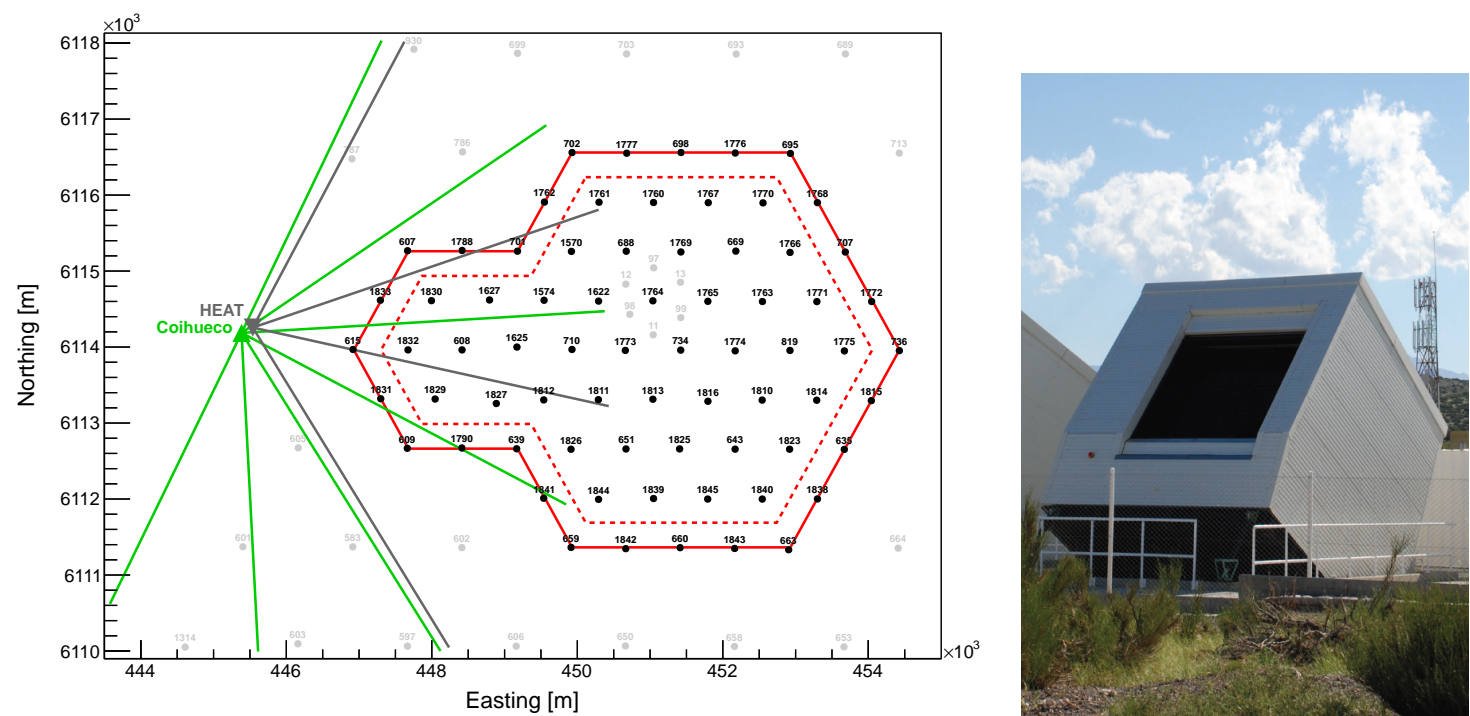

Figure 3: Left: layout of the dense $750 \mathrm{~m}$ array in the field of view of the FD builds Coihueco and HEAT. Right: Picture of one HEAT telescope with the field of view inclined by $30^{\circ}$
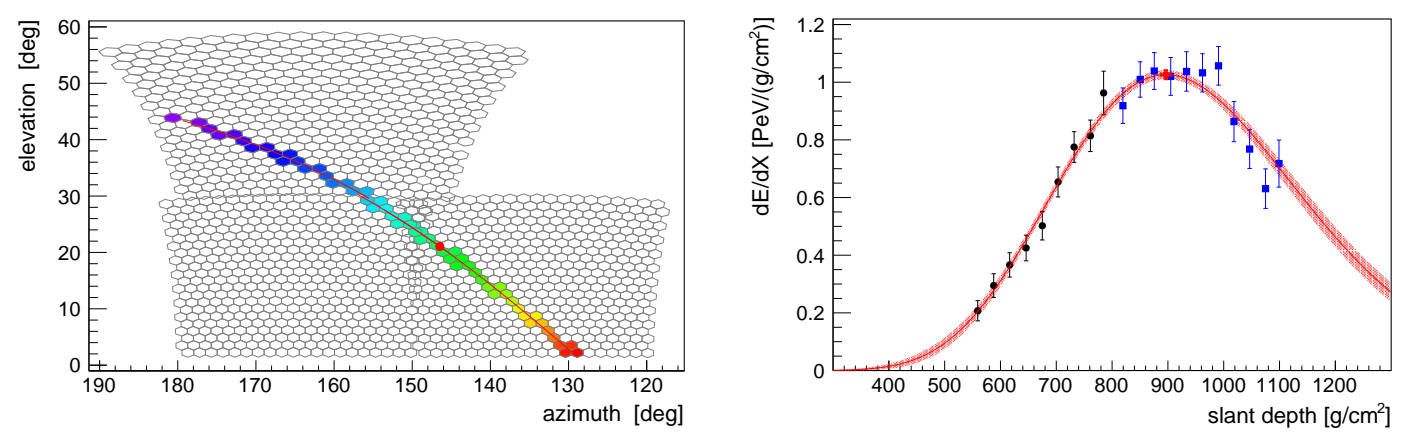

Figure 4: Example of a simulated photon with the energy of $0.5 \mathrm{EeV}$. Left: the camera view with the color encoding the timing form early (blue) to late (red). Right: the longitudinal profile measured by HEAT (black circles) and Coihueco (blue squares) with the Gaisser-Hillas fit (red line). The red cross indicates the $X_{\max }$ position.

that the mean is equal to zero and the standard deviation equal to one, resulting in $g L_{\mathrm{LDF}}$ and $g \Delta$. The PCA is performed on the burn sample of $2 \%$ of the Auger data and a part of the MC of about equal statistics. The PCA axis is shown in Fig. 1 right.

The selection cut for the signal has been fixed in [2] to an efficiency on the non-preshowering photon signal of 50\%. We keep this cut-value for consistency here, noting that at energies closely above $10 \mathrm{EeV}$ the background rejection is not guaranteed to be $100 \%$. Applying the analysis to the search data-set resulted in the distribution of the data and the photon MC in the PCA variable as shown in Fig. 2. In the tail of the data-distribution, 11 events are above the threshold, two of them at an energy above $20 \mathrm{EeV}$. The number of candidates evolved with respect to [3] due to the increase of exposure and slight changes in the reconstructed shower parameters. Further analysis using proton simulations for the geometry and energy of the candidates are not excluding the background hypothesis of the candidates. Here the large uncertainties of the hadronic interactions in the tail of 


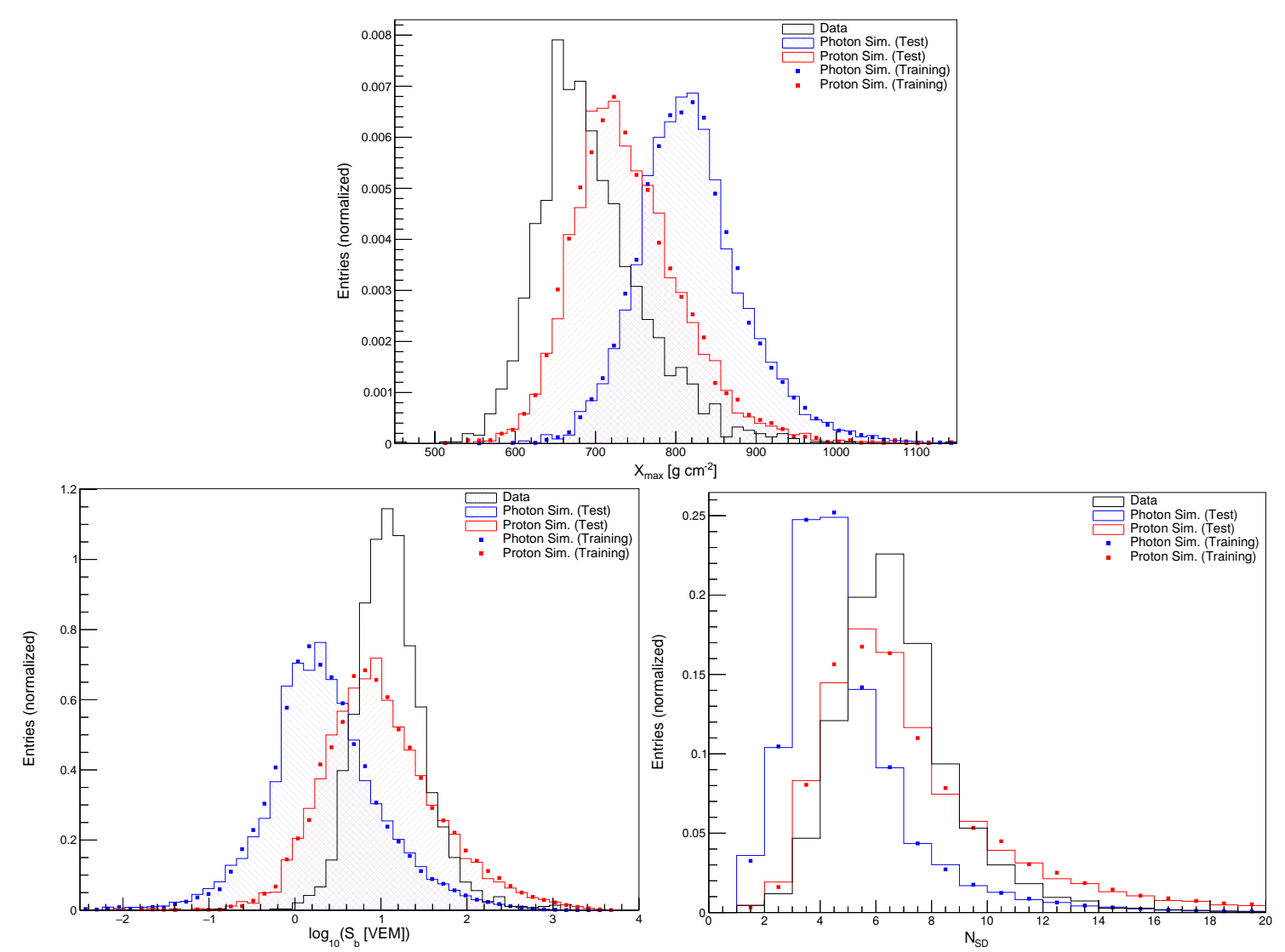

Figure 5: Observables used as input for the BDT as described in the text are shown for the photon signal MC (blue), the proton background MC (red), and the data-set (black).: $X_{\max }$ measured by FD (top), $S_{b}$ (bottom left) as well as $N_{\mathrm{SD}}$ (bottom right) as measured by SD.

the distributions limit the significance of such a test. As we can neither exclude the hypothesis of the candidates to be hadronic, nor can we prove the candidates to be photons, we conservatively determine the upper limit on the photon flux at $95 \%$ confidence level, as shown in Fig. 7. Therefore, though improving the exposure by almost a factor of two, the limit for the lower energy of $10 \mathrm{EeV}$ is background limited and even slightly worse than in [3]. For energies above $20 \mathrm{EeV}$ the limits are significantly improved due to the large increase in exposure while not obtaining any new candidate.

\section{Diffuse photon flux at lower energies}

At energies below $10 \mathrm{EeV}$ the number of SD stations with signal is too small to have a significant separation of photon-induced and nucleus-induced showers. But the increased event statistics allows to use the direct $X_{\max }$ measurement for the $15 \%$ uptime of the FD. We follow the measurment in [6] combining the $X_{\max }$ with the SD observables $S_{b}$ [20],

$$
S_{b}=\sum_{i}^{N_{\mathrm{SD}}} S_{i}\left(r_{i}\right)\left(\frac{r_{i}}{1000 \mathrm{~m}}\right)^{b}
$$




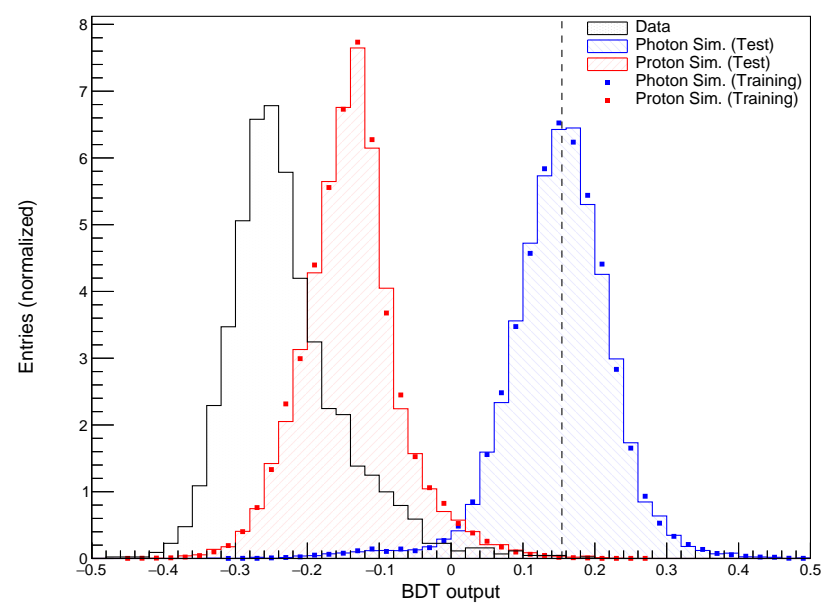

Figure 6: Output variable of the BDT trained on simulations together with the distributions for the photon signal MC (blue), the proton background MC (red), and the data-set (black).

with $b=4$ and the number of SD stations, $N_{\mathrm{SD}}$. Here, the measurment is extended towards energies below $1 \mathrm{EeV}$ by using data of the sub-array at smaller spacing of $750 \mathrm{~m}$ as shown in Fig. 3 and the measurment of $X_{\max }$ with the FD extension HEAT. The event selection is done according to [21]. MC simulations for photons and protons have been generated similar to Sec. 3. Using these as signal and background, a Boosted Decision Tree (BDT) has been trained. To take the energy and zenith angle dependence of the three discriminating observables into account they are used as additional inputs to the BDT. An example for a simulated photon event can be seen in Fig. 4. Before applying the BDT on the data it has been tested on a 5\% burn-sample subsequently excluded from the measurement. The data period used in this analysis is 1 Jun 2010 to 31 Dec 2015. The distributions of the input variables for the different MC samples and the data-sample are shown in Fig. 5. The separation power of the input variables is in this projection reduced due to their energy dependence. The resulting distribution of the BDT output variable is plotted in Fig. 6. Again, the selection cut on the BDT output variable is a-priori set to the median of the photon distribution, leading to a selection efficiency of 50\%. After application of the photon selection cut to the data sample only one candidate event is selected. This number is compatible with the background expectation from the proton MC. Evaluating the hybrid exposure for the given time-period, we determine the upper limits on the integral photon flux for the thresholds of $0.2,0.3,0.5$ and $1 \mathrm{EeV}$ at $95 \%$ confidence level. These flux limits are displayed together with the flux limits at the highest energy described in Sec. 3 in Fig. 7. They are compared to other measurements, partially derived at $90 \%$ confidence level.

\section{Conclusion}

With the increase in exposure at the highest energies and the extension towards lower energies using the enhancements of the Pierre Auger Observatory, the $750 \mathrm{~m}$ SD array and the FD HEAT, most stringent limits on the photon flux can be achieved. In combination with [6], the measurments presented here form the most sensitive UHECR photon search over about three orders of magnitude starting at $0.2 \mathrm{EeV}$. 


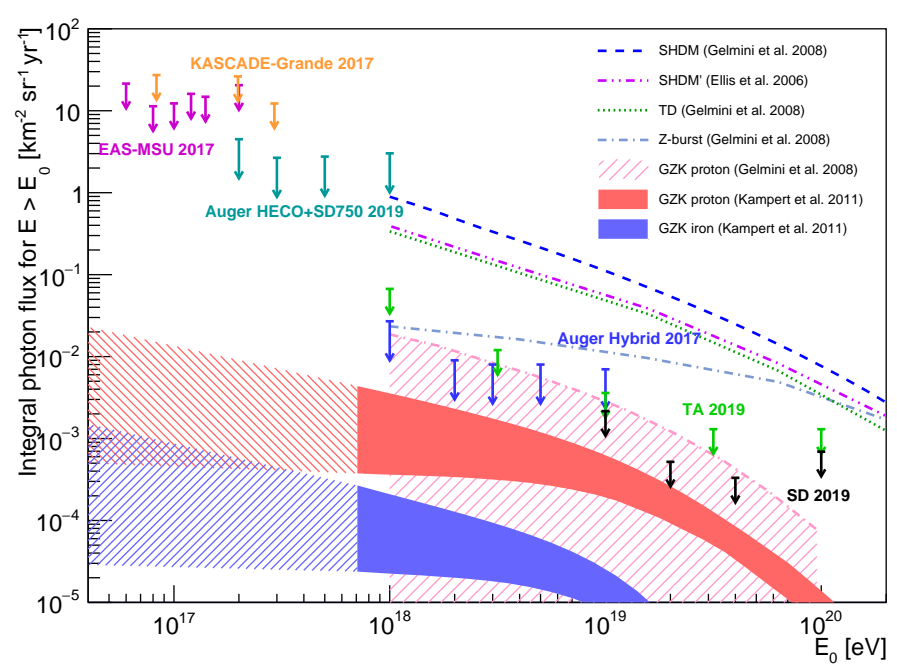

Figure 7: Photon flux limits at 95\% C.L. for the different analysis of the Pierre Auger Observatory, compared to model predictions $[14,15,16]$ and other experimental limits at 95\% C.L. [17], as well as at 90\% C.L. [18, $19]$.

\section{References}

[1] A. Aab [Pierre Auger Collaboration], Nucl. Instrum. Meth. A 798 (2015) 172.

[2] The Pierre Auger Collaboration, Astropart. Phys. 29 (2008) 243 [arXiv:0712.1147].

[3] C. Bleve for the Pierre Auger Collaboration, PoS(ICRC2015)1103, 2015.

[4] The Pierre Auger Collaboration, Astropart. Phys. 27 (2007) 155 [arXiv:astro-ph/0606619].

[5] The Pierre Auger Collaboration, Astropart. Phys. 31 (2009) 399 [arXiv:0903.1127].

[6] The Pierre Auger Collaboration, JCAP 04 (2017) 009 [arXiv:1612.01517].

[7] The Pierre Auger Collaboration, ApJ 789 (2014) 160 [arXiv:1406.2912].

[8] The Pierre Auger Collaboration, ApJL 837 (2017) L25 [arXiv:1612.04155].

[9] D. Heck et al., Report FZKA 6019 (1998).

[10] T. Pierog et al., Phys. Rev. C 92 (2017) 034906 [arXiv:1306.0121].

[11] P. Homola et al., Comput. Phys. Comm. 173 (2005) 71 [arXiv:astro-ph/0311442].

[12] P. Billoir et al. arXiv:astro-ph/0701583.

[13] The Pierre Auger Collaboration, Phys. Rev. D 96 (2017) 122003 [arXiv:1710.07249].

[14] G. Gelmini, O. Kalashev, D. Semikoz, JETP 106 (2008) 1061 [arXiv:astro-ph/0506128].

[15] J. Ellis, V. E. Mayes, D. V. Nanopoulos, Phys. Rev. D 74 (2006) 115003 [arXiv:astro-ph/0512303].

[16] K.-H. Kampert et al., Proceedings ICRC 2 (2011) 198.

[17] Telescope Array Collaboration, Astropart. Phys. 110 (2019) 8 [arXiv:[arXiv:1811.03920].

[18] KASCADE-Grande Collaboration, ApJ 848 (2017) 1.

[19] Yu. Fomin et al., Phys. Rev. D 95 (2017) 123011.

[20] G. Ros et al., Astropart. Phys. 35 (2011) 140 [arXiv:1104.3399].

[21] J. Bellido for the Pierre Auger Collaboration, PoS(ICRC2017)506, 2017. 